\title{
Improved PID Friction Feed-forward Compensation Control Based on Segment Friction Model
}

\author{
Pu Yang ${ }^{*}$, Zecheng Zhang, Jing Zhao and Dake Zhou
}

\author{
College of Automation Engineering, Nanjing University of Aeronautics and Astronautics, Nanjing, 210016, China
}

\begin{abstract}
An improved PID friction feed-forward compensation control based on segment friction model is presented for nonlinear friction perturbation problems of high precision optoelectronic tracking stable platform systems. From the view of engineering application, the friction characteristics of stable platform servo systems are considered, a simple and reasonable segment friction model is established, and a practical model for parameter identification is given. According to this model, improved PID friction feed-forward compensation control is designed. Through the feed-forward compensation, friction disturbance effect on system stability is reduced, and low-speed performance of the system is improved. By introducing the improved PID control, both integral saturation and peak jitter of system speed output are eliminated, and system control performance is improved. Finally, through the analysis and comparison of practical stable platform experiments, the rationality and effectiveness of the design method is proved.
\end{abstract}

Keywords: Stable platform, friction compensation, feed- forward control, PID control.

\section{INTRODUCTION}

In optoelectronic servo systems, in order to overcome steady state error and low-speed instability, brought by disturbance moments of nonlinear friction, one of the most direct way is to reduce system friction moments as much as possible by improving the accuracy of moving parts as well as lubrication [1]. However, this approach will cost a lot, and it is impossible to eliminate system nonlinear friction completely. In high precision optoelectronic tracking servo mechanism, owing to the necessity of dynamic sealing, great influence of friction moments exist. Therefore, control strategy with friction compensation is an effective and saving way which can further reduce the friction nonlinear influence [2]. Friction compensation methods are divided into two classes: some are model dependent friction compensation methods [3-5], while others are model independent friction compensation methods [6-8]. The friction compensation methods based on model are earlier researched and good for the practical application. This kind of methods need suitable mathmatic models of friction to forecast the value essentially, so that control system can compensate the friction. Therefore, the application effect of this kind of methods depends on the precision of the friction established models.

In this paper, an improved PID friction feed-forward compensation control method based on segment friction model is put forward for the friction disturbance problems in optoelectronic stable platform systems, effectively increasing the precision of friction compensation model, suppressing friction disturbance, and improving the robustness of the control systems.

\section{BASIC IDEA AND STRUCTURE OF FRI-CTION FEED-FORWARD COMPENSA-TION}

Traditional compensation structure based on friction model uses the feedback form shown in Fig. (1a). The shortcoming of this structure is that the inputs of friction model are the measured feedback speeds, which will lead to a sampling period delay in some practical heavy-loaded optoelectronic servo digital control systems. Meanwhile, feedback control loop comprised of the friction moments feedback compensation with intrinsic nonlinearities, may cause instability of control system [9]. Therefore, friction feed-forward compensation structures shown in Fig. (1b) is used.

In Fig. (1b), it has

$G_{q}(s)=\frac{W_{v}(s) G_{m}(s)}{1+W_{v}(s) G_{m}(s)}$

The speed input of friction model is estimated speed obtained from the reference input, and the feed-forward compensation will not change the stability of servo system, so as to avoid all disadvantages shown in feedback of Fig. (1a). In the stable platform servo control system, the friction moments in system motion can be offset by superimposing a feed-forward compensation signal related to the friction moments on the output signal of system closed-loop controller based on this friction feed-forward compensation structure. Therefore, the establishment of a simple and reasonable friction model is the first step of the design of friction feedforward compensation control method. 


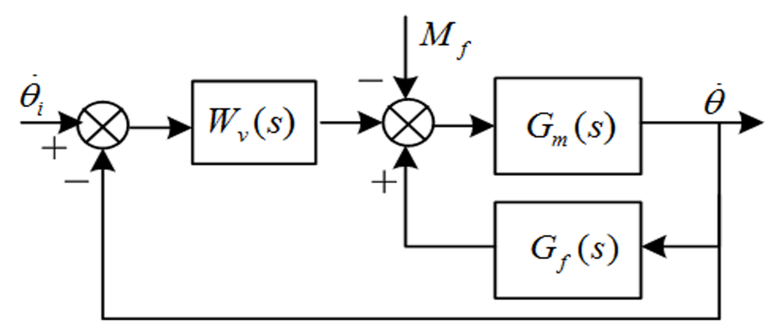

(a) friction feedback

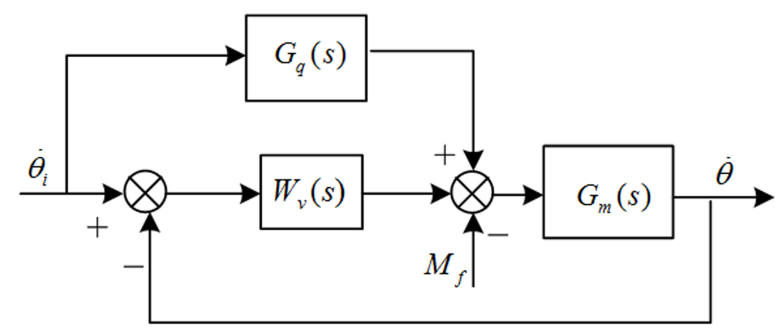

(b) friction feed-forward

Fig. (1). Compensation structure based on friction model.

\section{ESTABLISHMENT OF SEGMENT FRICTION MODEL IN STABLE PLATFORM SYSTEM}

The friction characteristics of general servo system is very complex, however, from the view of engineering application, the friction can be divided into two zones based on usual sliding circumstances before and after the start of optoelectronic tracking stable platform systems: static friction zone and dynamic friction zone, known as pre sliding zone and sliding zone [10].

\section{A. 3.1. Model Structure and Parameter Identification of Static Friction Field}

The control inputs used for friction compensation are added into the control system as estimates of friction moments, and mutation of static-dynamic friction will cause oscillation when the system is at low speed from pre sliding stage to sliding stage. So the friction compensation torque input should be able to avoid the discontinuity caused by torque mutation. Combined with the characteristics of friction in static friction zone stable platform servo system used in experiment, Sigmoid function ( $S$ function) is chosen as friction model structure of static friction zone [11], the general expression is:

$$
f(t)=a+\frac{b}{1+e^{-c t}}
$$

Where $a, b, c$ are constants.

Let us take $t_{0}$ as moment when the speed is zero, $t_{y h}$ as interval between $t_{0}$ and the moment when macroscopic motions occur in stable platform shafts. So when the stable platform moves one-way, $t_{0}$ refers to the moment when the working speed of stable platform is zero and the systems just issue the start command. When the stable platform moves two-way, $t_{0}$ refers to the moment when movement of stable platform changes and passes through the zero speed point. So, in the time interval $\left[t_{0}, t_{0}+t_{y h}\right]$, speeds of each frame of stable platform are zero. However, there is elastic deformation in the transmission parts between the motor and the load though there is no movement in the stable platform. In pre sliding stage of friction, friction moments can be seen as functions only related to time.

For the convenience of calculation and analysis, only the forward motions of the stable platform are considered, similar in negative situation. Let us set $t_{0}=0$, then the transition time of friction torque from pre sliding stage to sliding stage is $\left[0, t_{y h}\right]$. According to the equation (1), the stable platform friction model in static friction zone can be established:

$$
M_{s t a}(t)=k_{1}+\frac{k_{2} f_{\max }}{1+e^{-k_{3} t}}
$$

Where, $f_{\max }$ represents the maximum static friction moment, $k_{l}$ is scaling factor, which is used to ensure continuity for friction moment in the sliding stage and dynamic friction zone, $k_{2}$ is friction moment regulator, and $k_{3}$ is curve slope. In practical application, each parameter of model (2) is determined in the following way.

In the equation (1), let us set $a=0, b=1$, then:

$$
f(t)=\frac{1}{1+e^{-c t}}
$$

Let us set a constant $f_{y h}, 0<f_{y h}<1$, to make $f\left(t_{y h}\right)=f_{y h}$, then: 
$c=\frac{1}{t_{y h}} \ln \frac{f_{y h}}{1-f_{y h}}$

Curve slope of model (2) is chosen as $k_{3}=\mathrm{c}$. In order to avoid the mutation of friction torque after entering the sliding stage, equation (2) should satisfy $M_{s t a}(0)=0$ and $M_{s t a}\left(t_{y h}\right)$ $=f_{\max }$. Therefore, the equations can be gotten:

$\left\{\begin{array}{l}k_{1}+\frac{k_{2} f_{\max }}{2}=0 \\ k_{1}+\frac{k_{2} f_{\max }}{1+e^{-c t_{y h}}}=f_{\text {max }}\end{array}\right.$

By solving equations (5), it can be obtained:

$k_{1}=\frac{f_{\max }}{1-2 f_{y h}}, k_{2}=\frac{2}{2 f_{y h}-1}$

In the equation (6), the maximum static friction moment $f_{\max }$ is determined by experiment, the specific method is given in the design of dynamic friction model.

Overall, by the equation (4) and (6), all parameters of the friction model in static friction zone (2) can be calculated. In practical application, the friction model of the system in the static friction field can be established only by setting a constant $f_{y h}$ according to control performance requirement and friction characteristics of the system. Besides, when friction model reference trajectory needs to be changed, only the model curve slope $k_{3}$ needs to adjust, and there is no need to change other parameters.

\subsection{The Model Structure and Parameters Identification of Dynamic Friction Zone}

In the slip stage of the systems, friction moments of stable platform servo systems are functions related to speed. The relationship between the friction moments and steadystate speeds usually expresses as Stribeck curve [12], so the friction model of dynamic friction zone is described directly by equation (7):

$$
M_{\text {dyna }}(\omega)=\left[f_{k}+\left(f_{\max }-f_{k}\right) e^{-\left(\omega / \omega_{s}\right)^{2}}+m_{v}|\omega|\right] \operatorname{sgn}(\omega)
$$

Where, $f_{k}$ is the Coulomb friction moment, $f_{\max }$ is the maximum static friction moment, $m_{v}$ is viscous friction coefficient, $\omega$ is instantaneous velocity system, $\omega_{s}$ is the critical speed of Stribeck, and it is the Stribeck parameter that reflects the friction moment decreases according to the index rate from static friction to dynamic friction.

There are four unknown parameters in the Stribeck friction model. In the practical application, determination methods of each parameter are as follows:

Parameter 1: (the maximum static friction $f_{\max }$ ) by the equation (7), let us set the instantaneous speed $\omega=0$, the following can be obtained:

$M_{\text {dyna }}(0)=f_{\max }$
Although it is easy to determine the value of parameter $f_{\max }$ in theory, in the practical application process, we can find that friction moment data is different from what Stribeck model describes when the speed is very small. The main reason is the Stribeck friction model only describes the dynamic friction characteristics of friction zone, neglecting the friction in pre slip zone. Therefore, in practice, maximum static friction moment $f_{\max }$ can be determined by the experiment. Firstly, the stable platform should work at the mode of speed servo testing, control voltage of torque machine is increased until the stable platform control frame has macroscopic motion in the control program, the system control output data recorded at this moment can be calculated to get the maximum static friction moment. In the experiment, attention should be paid to that, the maximum static friction torques of positive and negative direction motion in each shaft are different. Therefore, They need to be tested respectively.

Parameter 2 and 3: (Coulomb friction $f_{k}$ and viscous friction coefficient $m_{v}$ )when $\omega \gg>\omega_{s}$, it can be gotten from equation (7):

$M_{\text {dyna }}=f_{k}+m_{v} \omega$

Obviously, the equation (9) represents a line whose slope is $m_{v}$. The intersections of the curve and the longitudinal axis of the friction torque represents the value of $f_{k}$. Therefore, the friction torque data of stabilized platform in high speed running stage is collected through experiment, a straight line is fitted by processing. Then according to the equaiton (9) values of Coulomb friction $f_{k}$ and viscous friction coefficient $m_{v}$ can be drawn.

Parameter 4: (critical Stribeck speed $\omega_{s}$ ), let us set $\omega=\omega_{s}$, from equation (7) (4.59), it has:

$$
\begin{aligned}
M_{\text {dyna }}\left(\omega_{s}\right) & =f_{k}+\left(f_{\max }-f_{k}\right) e^{-1}+m_{v} \omega_{s} \\
& \approx 0.3679 f_{\max }+0.6321 f_{k}+m_{v} \omega_{s}
\end{aligned}
$$

Similarly, equation (10) represents a line whose slope is $m_{v}$ as well, the intersections of this line and the longitudinal axis is $f_{m s}=0.3679 f_{\max }+0.6321 f_{k}$, and it intersects with the Stribeck speed friction torque curves at the point $\omega=\omega_{s}$. So, the intersection point of two lines is determined by mapping, the parameters $\omega_{s}$ can be identified according to the abscissa of this point.

Synthesize friction model of static friction zone and slip zone in subsections $A$ and $B$. Friction model of stable platform system can use a segment model to represent:

$M_{f}(t)=\left\{\begin{array}{lr}M_{s t a}(t), & 0 \leq t \leq t_{y h} \\ M_{\text {dyna }}(\omega), & t>t_{y h}\end{array}\right.$

By equation (11), in practical control, transition time $t_{y h}$ that systems move from static friction zone to dynamic friction zone also need to be identified, to distinguish critical point between static and dynamic friction zone and complete 
switch of friction models. The conclusion can be got through the theoretical analysis in [13], that the transition time is inversely proportional to the square root of the system initial stage acceleration. Accordingly, in practical design, this transition time $t_{y h}$ is calculated and adjusted real-time based on the stabilization platform output of speed controller at start-up moment:

$t_{y h}=\lambda_{J}\left(u_{i n}\right)^{-1 / 2}$

Where, $\lambda_{J}$ is the adjustment factor, $u_{i n}$ is the system speed controller output at start-up time.

\section{DESIGN OF IMPROVED PID CONTROL WITH NONLINEAR ACCELERATION FEEDBACK}

The basic theory of PID control is to combine the $e(\mathrm{t})$, which represents the bias between controller and reference input, and its integration, and its differentiation linearly. Then the control input signal $u_{\text {pid }}(\mathrm{t})$ can be generated. The ideal digital PID control equation after discretization is:

$u_{p i d}(n)=K_{p} e(n)+K_{i} \sum_{j=0}^{n} e(j) T+K_{d}[e(n)-e(n-1)] / T$

Where, $T$ is the sampling period, $K_{p}$ is scaling coefficient, $K_{i}$ is integral coefficient, $K_{d}$ is differential coefficient, $u_{p i d}(\mathrm{n})$ is control volume at moment $n T$, and $e(\mathrm{j})$ is the error at moment $j T$.

When the traditional PID control(13) is used for stable platform servo speed control, there will be the phenomena of integral saturation and the speed chattering. Those phenomena take place because that: when the system target suddenly changes greatly, traditional PID differential output increases or decreases rapidly; when the system is in the junction of dynamic and static friction, and gyro speed output is very low, if the load fluctuates or model parameters change, it will make the gyro feedback signals accelerate and then cause the speed peak.

In order to suppress the above adverse influence factors and improve the system control performance, the traditional PID control is improved in the practical application. Firstly, PID control [14, 15] with variable speed integral and incomplete differential is used. The idea of variable speed integral is setting an integral coefficient in the integral according to deviation and taking advantage of the slowly vary of system to change the integral output. The variable integral coefficient $\alpha_{i}(k)$ is:

$\alpha_{i}(k)=\left\{\begin{array}{cl}1 & |e(k)| \leq \lambda_{1} \\ \frac{\lambda_{2}-e(k)}{\lambda_{2}-\lambda_{1}} & \lambda_{1}<|e(k)|<\lambda_{2} \\ 0 & |e(k)| \geq \lambda_{2}\end{array}\right.$

Where, $\alpha_{i}$ changes in interval $[0,1]$, and $\lambda_{1}, \lambda_{2}$ is the error threshold.
Then the PID control integral output is:

$u_{i}(n)=k_{i}\left[\sum_{j=0}^{n-1} e(j)+\alpha_{i}(n) \cdot e(n)\right] \cdot T$

Incomplete differential PID is that adding a first-order inertia to the original differential part, to ensure the differential function is gradual decline, thus system oscillation is avoided when the input value mutates. Let us set the time constant of first-order inertial $T_{f}$, the PID differential output will be:

$u_{d}(n)=\frac{k_{d}}{T}\left(1-\alpha_{d}\right) \sum_{j=1}^{n}\left[\alpha_{d}^{n-i} \Delta e(j)\right]+\alpha_{d}^{n} u_{d}(0)$

Where, $\alpha_{d}=\frac{T_{f}}{T_{f}+T}, u_{d}(0)$ is initial value of the differential output $u_{d}(n)$.

Secondly, gyro signal acceleration negative feedback is added into the PID control output, to increase system damping and minimize jitter. But the common linear acceleration negative feedback, will cause the decrease of response time of system output response. So in this system, the nonlinear acceleration negative feedback is designed. When stabilized platform is at low speed operation, acceleration negative feedback compensation value is adjusted according to different acceleration. The calculation equations are as follows:

$u_{g}=\left\{\begin{array}{cl}\tau_{g} a-a_{g} \operatorname{sgn}(a), & \left|\tau_{g} a\right| \geq a_{g} \text { and } \omega \leq \omega_{g} \\ 0, & \left|\tau_{g} a\right|<a_{g} \text { or } \omega>\omega_{g}\end{array}\right.$

Where, $a$ and $\omega$ are the gyro feedback acceleration value and speed value, $\tau_{g}$ is the adjustment factor, $a_{g}$ is the acceleration boundary value, $\omega_{g}$ is the speed boundary value.

Through equation(17) we can see that, when the speed of stable platform is small and the acceleration value is large, acceleration feedback is added into output controller, to increase system damping, and prevent the spike jitter; while, when the speed is relatively large or acceleration value is not big, the acceleration feedback compensation is canceled, to ensure the response speed of system.

Synthesize equation (13) (17), improved PID controller algorithm can be obtained :

$$
\begin{aligned}
u_{p i d}(n)=K_{p} e(n)+k_{i}\left[\sum_{j=0}^{n-1} e(j)+\alpha_{i}(n) \cdot e(n)\right] \cdot T \\
+\frac{k_{d}}{T}\left(1-\alpha_{d}\right) \sum_{j=1}^{n}\left[\alpha_{d}^{n-i} \Delta e(j)\right]+\alpha_{d}^{n} u_{d}(0)-u_{g}
\end{aligned}
$$

In practical application, parameters of the PID controller are roughly tuned by using critical proportioning method. Then it will be fine tuned according to the control result. Fig. (2) shows the structure diagram of improved PID friction feed-forward compensation control based on segment friction model. 


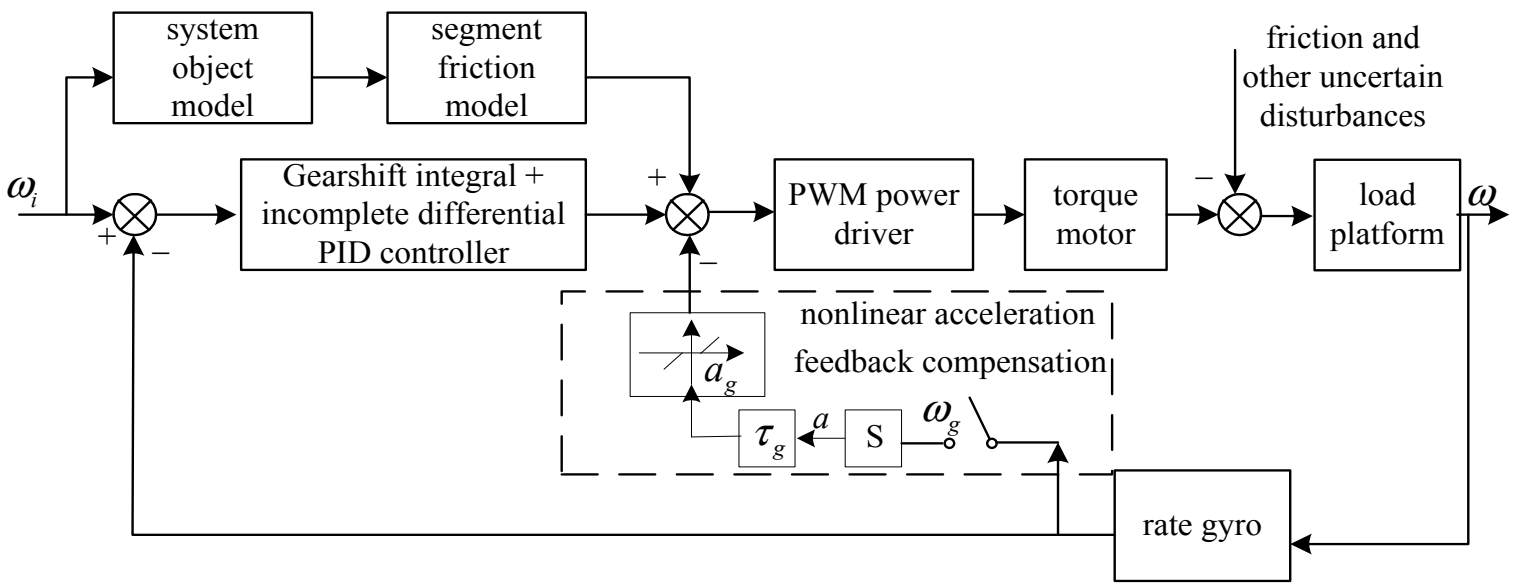

Fig. (2). Structure diagram of improved PID friction feed-forward compensation control based on segment friction model.

Table 1. Segment friction model parameter.

\begin{tabular}{|c|c|c|c|c|}
\hline & $\begin{array}{c}\text { Maximum static Friction } \\
\text { Torque } f_{\max }(\mathbf{N m})\end{array}$ & $\begin{array}{c}\text { Coulomb Friction Torque } \\
f_{k}(\mathbf{N m})\end{array}$ & $\begin{array}{c}\text { Critical Stribeck Speed } \\
(\mathbf{r a d} / \mathbf{s})\end{array}$ & $\begin{array}{c}\text { Viscous Friction Coefficient } \\
m_{v}(\mathbf{N m s} / \mathbf{r a d})\end{array}$ \\
\hline \hline Machine lockwise rotation & 0.286 & 0.107 & 0.002 & 0.025 \\
\hline $\begin{array}{c}\text { Machine counter-lockwise } \\
\text { rotation }\end{array}$ & -0.359 & -0.184 & -0.003 & 0.031 \\
\hline
\end{tabular}

\section{EXPERIMENT RESULTS AND ANALYSIS OF FRICTION COMPENSATION SPEED CONTROL}

The speed control systems of three shafts in a certain type optoelectronic tracking stable platform are tested respectively, the results are similar. In this paper, only the elevation shafts are considered, the experiment results of different methods are given. The speed controller is tested respectively by two kinds of methods, which are traditional PID control and improved PID friction feed-forward compensation speed control based on segment friction model (FCPID). Where, FCPID control identify model parameters according to segment friction model parameter identification method given in the second subsection. The results are shown in Table 1 .

Experiment 1: (low speed tracking experiment) Speed square wave input signal, whose amplitude is $0.2 \%$ and cycle is $360 \mathrm{~ms}$, is given, to test closed-loop and low-speed tracking performance of elevation shafts speed.

In Fig. (3), when the PID control is used, speed tracking performance of gyro speed closed-loop is not good, there is obvious "dead-zone" crawl and low-speed spike jitter at low speed, curve overshoot is large, and there exists obvious tracking error. Besides, the shaft tracking performance are different in positive and negative movement, denoting that there is change in friction disturbance parameter when each shaft is in different states.

After using FCPID control method, from Fig. (4) we can see, the curve fluctuation is significantly smaller. There is no dead zone in the curve transition process, no spike at low speed and no big overshoot at dynamic process. When it is tracking in positive and negative direction, the steady-state error decreases obviously, steady-state errors of positive and negative direction maintain in $0.05 \%$, and response time is less than $15 \mathrm{~ms}$. It is denoting that, the designed segment friction model can effectively compensate the friction torque disturbance of two direction motion, and effectively improve static and dynamic characteristics of the system low-speed stable tracking.

Experiment 2: (minimum smooth-speed slope tracking experiment) Position slope input signal $0.01 t$ is given, speed closed loop respectively uses the two kinds of control above, to test stabilized platform position tracking output curve.

By comparing Fig. (5) and Fig. (6), it can be known that, the fluctuation of position tracking output curve in low speed is significantly higher than the one of FCPID method. At this tracking speed, the output curve of FCPID control keeps smooth. The position error is limited in 10" according to the definition of the minimum smooth speed. By adjusting the slope signal, we can get that the system lowest smooth speed of above three kinds of control is about $0.0328^{\circ} / \mathrm{s}, 0.0043^{\circ} / \mathrm{s}$.

Experiment 3: (disturbance isolation test experiment) The position inputs of elevation shafts are given as zero, the speed loop uses the two kinds of control above respectively. System position output curve is tested by sinusoidal disturbances exerting on the stabilized platform system by using the base, whose amplitude is within $10^{\circ}$ and frequency is about $1 \mathrm{~Hz}$. 


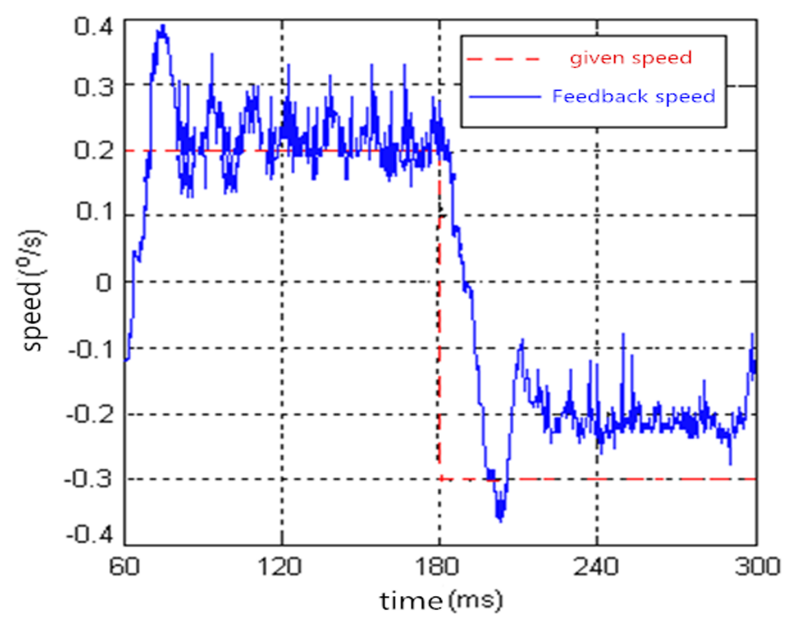

(a) Speed output curve

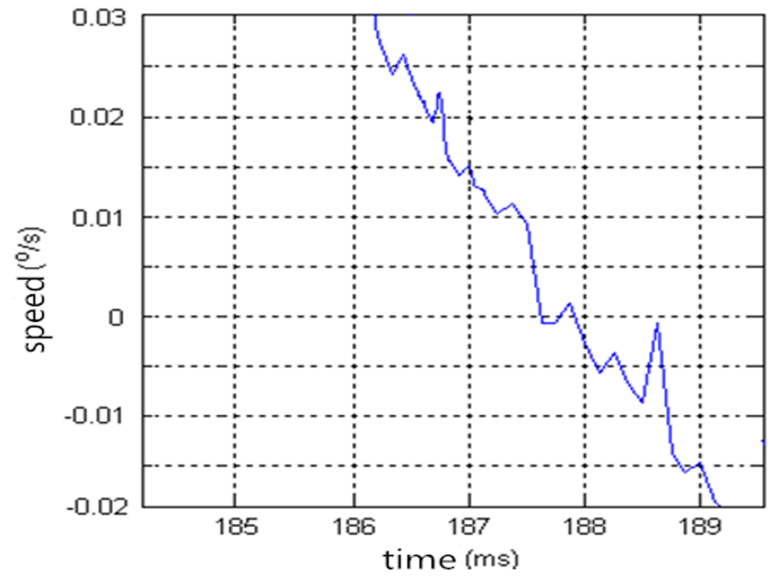

(b) Partial enlarged picture

Fig. (3). PID control low-speed tracking speed output curve.

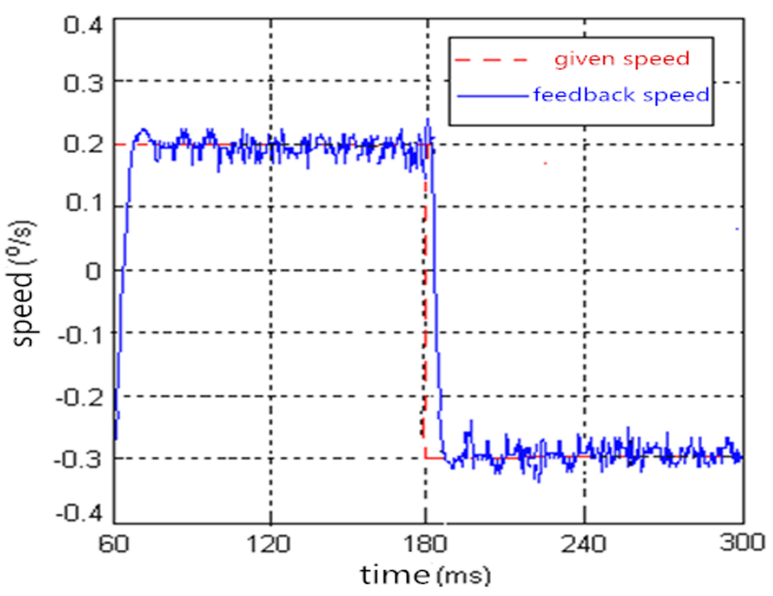

Fig. (4). FCPID control low-speed tracking speed output curve. 


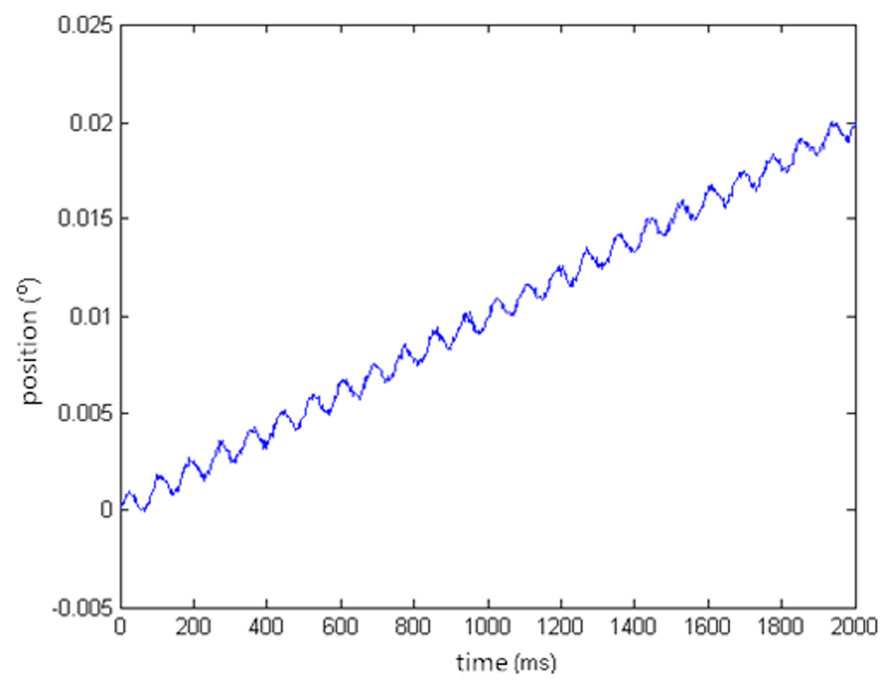

Fig. (5). PID control slope tracking position output curve.

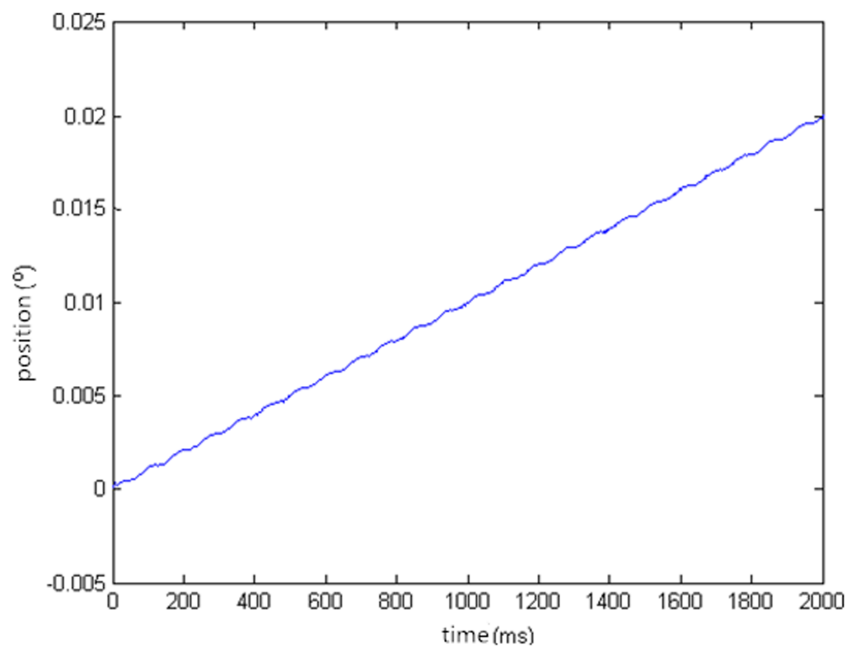

Fig. (6). FCPID control slope tracking position output curve.

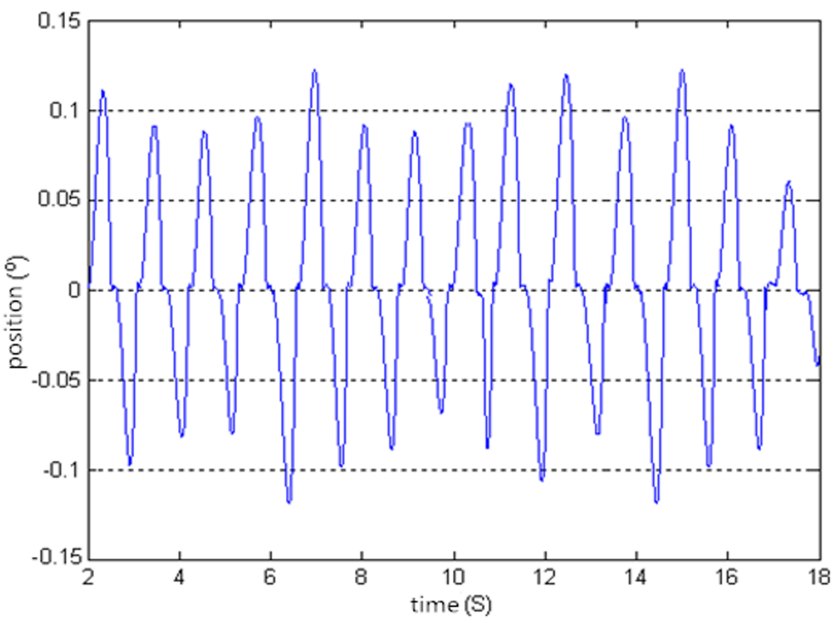

Fig. (7). PID control disturbance isolation curve.

By comparing Fig. (7) and Fig. (8), it can be known that, maximum error of instantaneous position declines from about $0.125^{\circ}$ originally to less than $0.08^{\circ}$. Compared with PID control, when the FCPID control is used, speed servo 


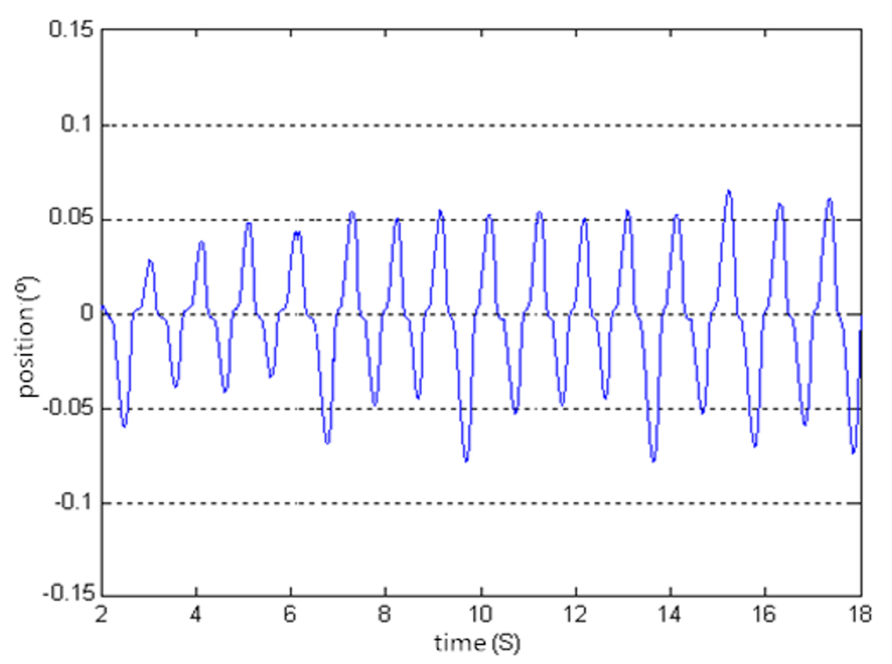

Fig. (8). FCPID control disturbance isolation curve.

system has higher disturbance isolation precision. According to the definition of disturbance isolation of inertial platform, we can get the disturbance isolations under control of three kinds of methods are: $-38.1 \mathrm{~dB}$ and $-44.7 \mathrm{~dB}$ by calculating the maximum error angle. Thus, using the friction feedforward compensation method designed in this paper can effectively improve the stability precision of the stable platform servo control systems.

\section{CONCLUSION}

In optoelectronic stable platform systems, the nonlinear friction disturbance is one of the key factors affecting the low speed performance of the stable platform. In order to improve the stability of stable platform at low speed, and increase tracking precision of the speed closed-loop, the friction compensation control method is neccessary. In this paper, segment friction mode of stable platform servo systems is established by using the experiment method to reduce the effect of optoelectronic control systems from friction disturbance, improve the system performance in low speed. On this basis, a kind of improved PID friction feed-forward compensation control based on segment friction model is designed. Not only it can ensure stability of friction compensation control systems, but also it can restrain the friction disturbance effectively. The tests on stable platform have showed that: compared with pure PID control, the friction feed-forward compensation methods effectively eliminate the dead-zone fluctuation of stable platform at low speed tracking, obtain the smaller lowest smooth speed, improve the control performance of the stable platform speed closedloop, and increase anti-interference performance of stable platform.

\section{CONFLICT OF INTEREST}

The authors confirm that this article content has no conflicts of interest.

\section{ACKNOWLEDGEMENTS}

This work is supported by the National Natural Science Foundation of China (Grant No. 61203090 and No.611 $72135)$, the Natural Science Foundation of Jiangsu Province of China (No.BK2012384) and the Fund of National Engineering and Research Center for Commercial Aircraft Manufacturing (No.SAMC14-JS-15-053).

\section{REFERENCES}

[1] Liu J K, Er L J. Sliding mode controller design for position and speed control of flight simulator servo system with large friction $[\mathrm{J}]$. Journal of System Engineering and Electronics, 2003, 14(3): 59-62

[2] Yinhu Xi,Yuan Zhou, Wei Zhang, Junhong Mao. An experimental method for measuring friction behaviors of linear rolling guides $[\mathrm{J}]$. Chinese Science Bulletin, 2014, 59(2930):3912-3918

[3] Yi-Cheng Huang and Mou-Sheng Lin. Tracking control of a piezo-actuated stage based on a frictional mode[J]. Asian Journal of Control, 2009, 11(3):287-294

[4] Hu Hongjie Wang, Yuanzhe and Sun Guowei. Hybrid Adaptive Compensation Control Scheme for High-Precision Servo System[J]. Transactions of Tianjin University. 2013, 19(3): 217-222

[5] Han S I, Lee K S. Sliding mode-based friction control with adaptive dual friction observer and intelligent uncertainty compensator[J]. Journal of Systems and Control Engineering, 2009, 1(08): 1129-1147.

[6] Tairen Suna, Hailong Peia and Yongping Pana,etc. Neural network-based sliding mode adaptive control for robot manipulators[J]. Neurocomputing, 2011, 74(14-15): 2377-2384

[7] Sunan Huang, Kok Kiong Tan. Intelligent Friction Modeling and Compensation Using Neural Network Approximations[J]. IEEE Transactions on Industrial Electronics, 2012, 59(8): $3342-3349$

[8] Wang, Yong Fu, Wang, Dianhui and Chai, Tian-You. Extraction and Adaptation of Fuzzy Rules for Friction Modeling and control Compensation [J]. IEEE Transactions on Fuzzy Systems, 2011, 19(4): 682-693

[9] Tsai M C, Chiu F I, Cheng M Y. Design and implementation of command and friction feed-forward control for $\mathrm{CNC}$ motion controllers[J]. IEEE Proceedings of Control Theory Application 2004, 151(1): 13-20

[10] Jun Young Yoon, David L. Trumpe. Friction modeling, identification, and compensation based on friction hysteresis and Dahl resonance $[\mathrm{J}]$. Mechatronics, 2014, 24(6):

[11] 734-741. 
[12] Park E C, Lim H, Choi C H. Characteristics and compensation of friction at velocity reversal [C]. Proceedings of the American Control Conference, 2001, 1: 582-587

[13] J. Le Rouzic, A. Le Bot and J. Perret-Liaudet, etc. FrictionInduced Vibration by Stribeck's Law: Application to Wiper Blade Squeal Noise[J].Tribology Letters, 2013, 49 (3):563572

[14] Park E C, Lim H, Choi C H. Position control of X-Y table at velocity reversal using presliding friction characteristics[J].
IEEE Transactions on Control Systems Technology, 2003, 11(1): 24-31

[15] Ji W, Li Q. Design study of adaptive fuzzy PID controller for LOS stabilized system[C]. Proceedings of the 6th International Conference on Intelligent Systems Design and Applications, 2006, 1: 336-341

[16] Huang Jian. Application of Variable Integral PID in AUV Heading Attitude Control[J]. Techniques of Automation and Applications, 2014, 33(5): 61-66

Received: September 16, 2014

(C) Yang et al.; Licensee Bentham Open.

This is an open access article licensed under the terms of the Creative Commons Attribution Non-Commercial License (http://creativecommons.org/licenses/by-nc/3.0/) which permits unrestricted, non-commercial use, distribution and reproduction in any medium, provided the work is properly cited. 